\title{
The relation between AGN hard X-ray emission and mid-infrared continuum from ISO spectra: Scatter and unification aspects ${ }^{\star} \star \star$
}

\author{
D. Lutz ${ }^{1}$, R. Maiolino ${ }^{2}$, H. W. W. Spoon ${ }^{3}$, and A. F. M. Moorwood \\ 1 Max-Planck-Institut für extraterrestrische Physik, Postfach 1312, 85741 Garching, Germany \\ 2 Osservatorio Astrofisico di Arcetri, Largo E. Fermi 5, 50125 Firenze, Italy \\ e-mail: maiolino@arcetri.astro.it \\ 3 Cornell University, Dept. of Astronomy, 219 Space Science Building, Ithaca, NY 14853-6801, USA \\ e-mail: spoon@isc.astro.cornell.edu \\ ${ }^{4}$ European Southern Observatory, Karl-Schwarzschild-Str. 2, 85748 Garching, Germany \\ e-mail: amoor@eso.org
}

Received 10 December 2003 / Accepted 26 January 2004

\begin{abstract}
We use mid-infrared spectral decomposition to separate the $6 \mu \mathrm{m}$ mid-infrared AGN continuum from the host emission in the ISO low resolution spectra of 71 active galaxies and compare the results to observed and intrinsic $2-10 \mathrm{keV}$ hard X-ray fluxes from the literature. We find a correlation between mid-infrared luminosity and absorption corrected hard X-ray luminosity, but the scatter is about an order of magnitude, significantly larger than previously found with smaller statistics. Main contributors to this scatter are likely variations in the geometry of absorbing dust, and AGN variability in combination with non-simultaneous observations. There is no significant difference between type 1 and type 2 objects in the average ratio of midinfrared and hard X-ray emission, a result which is not consistent with the most simple version of a unified scheme in which an optically and geometrically thick torus dominates the mid-infrared AGN continuum. Most probably, significant non-torus contributions to the AGN mid-IR continuum are masking the expected difference between the two types of AGN.
\end{abstract}

Key words. galaxies: active - galaxies: Seyfert - infrared: galaxies - X-rays: galaxies

\section{Introduction}

The mid-infrared and hard X-rays are two of the regions of the electromagnetic spectrum that are of particular interest for the study of active galactic nuclei (AGN). Hard X-rays, unless extremely obscured in fully Compton-thick objects, can provide a direct view to the central engine, and are often believed to be a reasonable isotropic measure of the bolometric luminosity of the AGN. The nuclear infrared continuum in Seyferts, in contrast, is due to AGN emission reprocessed by dust, either in the putative torus or on somewhat larger scales, e.g. inside the Narrow Line Region. The observed mid-infrared emission is thus a function not only of the AGN luminosity but also of the distribution of the obscuring matter and of the viewing direction of the observer. In the most simple form this is due to the covering factor and distance of the obscuring dust, but much

Send offprint requests to: D. Lutz, e-mail: lutz@mpe.mpg.de

* Based on observations with ISO, an ESA project with instruments funded by ESA member states (especially the PI countries: France, Germany, The Netherlands, and the UK) with the participation of ISAS and NASA.

$\star \star$ Table 1 is only available in electronic form at the CDS via anonymous ftp to cdsarc.u-strasbg.fr $(130.79 .128 .5)$ or via http://cdsweb.u-strasbg.fr/cgi-bin/qcat?]/A+A/418/465 more complex radiative transfer effects may occur in high optical depth configurations (e.g. Pier et al. 1992). In general terms, measurements of the mid-infrared continuum in conjunction with hard X-ray observations can be thought of as testing unification scenarios for AGN. A tight relation between the two quantities has recently been reported by Krabbe et al. (2001) on the basis of mid-infrared imaging of eight nearby Seyferts. Clavel et al. (2000) have found a large difference between the equivalent widths of the mid-infrared aromatic "PAH" emission features in type 1 and 2 Seyferts. Under the assumption that the type 1 and 2 subsamples are well matched in AGN luminosity and host properties, they interpret this as an orientation dependent depression of continuum in Seyfert $2 \mathrm{~s}$ with respect to the host-related isotropic $\mathrm{PAH}$ emission.

The issue is plagued, however, by the technical difficulty of isolating the AGN mid-infrared continuum from the host galaxy emission. IRAS has readily detected large numbers of AGN, but the host contribution to these large beam infrared spectral energy distributions (e.g. Spinoglio et al. 1995) is not easy to quantify and significant in all but the very powerful AGN. One way to address this difficulty is high spatial resolution imaging from groundbased telescopes, e.g. in the $L$ and $M$-bands (e.g. Alonso-Herrero et al. 2001) or the $N$-band (e.g. Maiolino et al. 1995; Krabbe et al. 2001). This method 
produces reliable results in cases of good surface brightness contrast between AGN and host, but can face ambiguities in cases where the AGN is surrounded by intense star formation on scales similar to the spatial resolution used, in particular if the observations are diffraction limited by a moderate size telescope (e.g. NGC 6240, NGC 4945; Krabbe et al. 2001).

We use the alternative approach of isolating the AGN midinfrared continuum spectrally, making use of the sizeable database of low resolution mid-infrared spectra of AGN that are a legacy of the Infrared Space Observatory ISO. Low resolution spectra of galaxies can be decomposed into three components (Laurent et al. 2000): a component dominated by the aromatic "PAH" features arising in photodissociation regions or the diffuse interstellar medium of the host, an HII region very small grain continuum rising steeply towards wavelengths beyond $10 \mu \mathrm{m}$, and for active galaxies a typically flatter thermal AGN dust continuum. Starlight is unimportant except for quiescent objects like ellipticals or nearby spirals with weak central star formation or AGN activity. The three components may also be obscured, with the additional complication of ice features (Spoon et al. 2002). A full spectral decomposition accounting for all these effects can be attempted in cases of good signal-to-noise ratio and full wavelength coverage (e.g. Tran et al. 2001; Spoon et al. 2004). Since most of our data are for the restricted ISOPHOT range (5.8 to $11.8 \mu \mathrm{m})$ that limits the accuracy of separating silicate absorption and $\mathrm{PAH}$ emission, and since some spectra are of limited $\mathrm{S} / \mathrm{N}$, we follow a more straightforward approach.

In the range covered by the ISOPHOT spectra, the AGN emission is most easily isolated shortwards of the complex of aromatic emission features (Laurent et al. 2000). We determine a continuum at $6 \mu \mathrm{m}$ rest wavelength, and eliminate non-AGN emission that will in many cases be present in the fairly large beam. This is done by subtracting a star formation template scaled with the strength of the aromatic "PAH" features arising in the host or in circumnuclear star formation. This method does not require to spatially resolve the AGN from the contaminating star formation, and will face its limits only when trying to identify a weak AGN in the presence of strong star formation or a stellar continuum that can be detectable for the most nearby galaxies. Our sample of 71 AGN is then used to quantify the relation of mid-infrared and Xray emission at significantly better statistics than previously possible.

\section{Data}

We have included in our sample those Seyferts or Quasars with low resolution spectra in the ISO archive for which hard $\mathrm{X}$-ray observations were available in the literature. Since our main goal is a comparison of thermal infrared emission with hard X-rays we have excluded objects believed to have a significant synchrotron contribution in the mid-infrared (e.g. Cen A, 3C 273). Of the Ultraluminous Infrared Galaxies for which a significant number of ISO spectra is available, we have restricted ourselves to a number of the brightest objects with clear and undisputed AGN contribution.

\subsection{Infrared spectroscopy}

Most of the spectra are from a rereduction of chopped ISOPHOT-S spectra of galactic nuclei in the ISO archive (Spoon et al. 2004, in preparation). The reduction was done using PIA ${ }^{1}$ version 9.0.1. Steps in the data reduction included: 1) deglitching on ramp level; 2) subdivision of ramps in four sections of 32 non destructive read-outs; 3) ramp fitting to derive signals; 4) masking of bad signals by eye-inspection; 5) kappa sigma and min/max clipping on remaining signal distribution; 6) determination of average signal per chopper plateau; 7) masking or correction of bad plateaux by eyeinspection; 8) background subtraction by subtracting off-source from on-source plateaux; 9) finally, flux calibration, using the signal dependent spectral response function. The absolute calibration is accurate to within $20 \%$. The ISOPHOT-S aperture is $24^{\prime \prime} \times 24^{\prime \prime}$ and in many cases includes noticeable host emission. A few spectra are based on different reductions. Circinus and NGC 1068 were observed in staring mode, and for NGC 4945 the ramp division of step 2) used only two sections. Mrk 1014 was reduced with the earlier PIA 7.2 and scaled to the PIA 9.0.1 flux scale. For NGC 1808 we have used an ISOCAM-CVF spectrum (Laurent et al. 2000 and priv. communication).

We derive the $6 \mu \mathrm{m}$ AGN continuum by a decomposition over a range from $5.5 \mu \mathrm{m}$ rest wavelength (or the minimum rest wavelength covered) to $6.85 \mu \mathrm{m}$. With the exception of the $6.2 \mu \mathrm{m}$ PAH feature (also associated with a continuum-like "plateau"), this region is relatively free of spectral structures or strong emission lines. Absorption features from ice $(6 \mu \mathrm{m})$ and hydrocarbons $(6.8 \mu \mathrm{m})$ are found in highly obscured objects but are mostly undetected in our AGN sample (see Spoon et al. 2002). We can thus fit the spectrum by the superposition of a star formation component dominated by the $6.2 \mu \mathrm{m}$ PAH feature and a simple linear approximation for the AGN continuum. For the star formation component, we use the SWS spectrum of M 82 (Sturm et al. 2000), rebinned to the exact sampling of the individual low resolution spectra. Figure 1 illustrates this using the example of NGC 7469 which is well known to host both a powerful type $1 \mathrm{AGN}$ and a strong circumnuclear star forming ring (e.g., Mazzarella et al. 1994; Genzel et al. 1995). The correction for "continuum" associated with the aromatic emission is relatively modest in this object, but more than half in some Seyferts in our sample. The results of the fit procedure are a $6 \mu \mathrm{m}$ continuum as illustrated in Fig. 1 and an average flux density of the PAH component over the rest wavelength range 6.1 to $6.35 \mu \mathrm{m}$, both including a $1+z$ correction so that the rest frame spectrum $v f_{v}$ matches the observed frame one.

The SWS spectrum of M 82 is technically well suited for this decomposition but represents just a single object that may be not fully representative for star forming objects in general, given a certain dispersion in relative importance of "PDR" and "HII" components in the sense of the model of Laurent et al. (2000). We have thus applied the decomposition also to a number of bona fide star forming galaxies (NGC 23, NGC 232, NGC 520, IC 342, NGC 3256, M 83, NGC 5653, NGC 6090,

1 PIA is a joint development by the ESA Astrophysics division and the ISOPHOT consortium. 

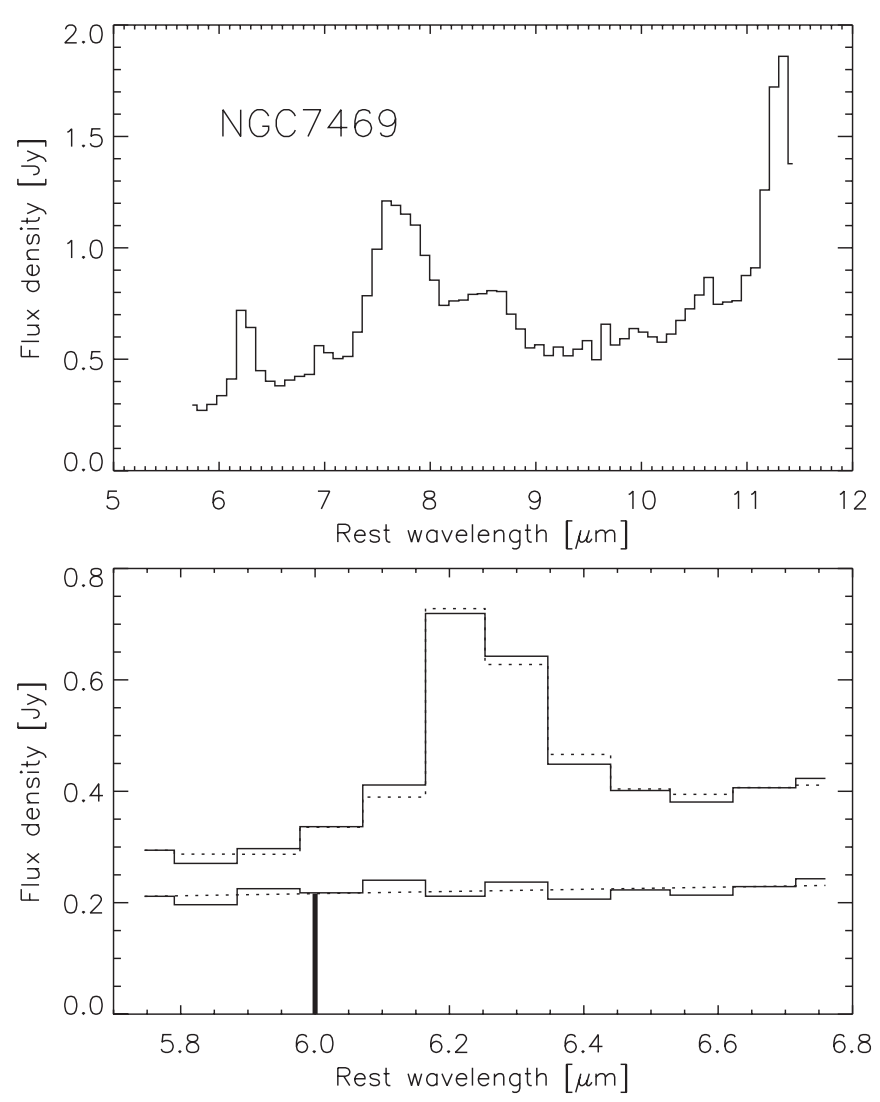

Fig. 1. Illustration of the decomposition used to isolate the AGN continuum. Top panel: ISOPHOT-S spectrum of the mixed AGN/starburst galaxy NGC 7469, showing strong PAH emission as well as an elevated continuum. Bottom panel: Cutout of the region around the $6.2 \mu \mathrm{m}$ PAH feature. Top continuous line $=$ observed spectrum. Top dotted line $=$ fit by the sum of the rebinned SWS M 82 PAH spectrum and a linear AGN continuum. Bottom dotted line = fitted AGN continuum. Bottom continuous line $=$ difference of observed spectrum and fitted PAH component. The thick vertical line indicates the AGN $6 \mu \mathrm{m}$ continuum, obtained by evaluating the fitted continuum at that wavelength. An additional correction is applied to this value to account for differences between M 82 and an average starburst.

NGC 6946, NGC 7252, NGC 7552), excluding starbursts with clear evidence for an additional minor AGN (e.g. NGC 3690, Della Ceca et al. 2002). Indeed, M 82 is found to have relatively low $6 \mu \mathrm{m}$ continuum compared to most of these objects. Averaging over all these star forming objects including M 82 we find a mean residual $6 \mu \mathrm{m}$ continuum which is 0.096 times the average 6.1 to $6.35 \mu \mathrm{m}$ PAH flux density, with a dispersion of 0.085 . We consider both this average value and the dispersion in our analysis of AGN. The $6 \mu \mathrm{m}$ AGN continua listed in Table 1 are the values obtained after subtracting from the direct fit result 0.096 times the average 6.1 to $6.35 \mu \mathrm{m}$ PAH flux density. Error estimates for the $6 \mu \mathrm{m}$ continuum are the quadratic sum of two components. The first is a measurement error based on individual pixel noise derived from the dispersion in the difference of observation and fit. The second is 0.085 times the average 6.1 to $6.35 \mu \mathrm{m}$ PAH flux density, thus considering the dispersion in the properties of the comparison star forming galaxies. Either component is found to dominate for part of the AGN sample, depending on $\mathrm{S} / \mathrm{N}$ and the relative importance of the PAHs. Limits quoted in Table 1 are $3 \sigma$ limits based on these considerations.

We ascribe the $6 \mu \mathrm{m}$ continuum derived in this way to an AGN. This is not strictly correct in all cases, as very intense star forming environments outside of the parameter range covered by our comparison objects also can produce a stronger continuum with relatively weak PAH at these wavelengths. This is true, for example, for the obscured region in SBS 0335-052 (Thuan et al. 1999), the mid-infrared peak in the Antennae (Mirabel et al. 1998), and likely the circumnuclear region of Arp 220 (Spoon et al. 2004). We consider this of minor importance, however, for our sample which does not include star forming dwarfs, and only some of the best established AGN among the ULIRGs.

In some of the weaker AGN in nearby galaxies, photospheric emission from the central old stellar population may contribute significantly to the measured $6 \mu \mathrm{m}$ continuum. The $6 \mu \mathrm{m}$ stellar continuum can be extrapolated from the stellar $K$-band continuum. From four ellipticals without PAH emission (NGC 3379, NGC 4374, NGC 2300, NGC 4649; Lu et al. 2003, Xilouris et al. 2004, S. Madden priv. comm.) we estimate a scaling $S_{6 \mu \mathrm{m}} \sim 0.19 \times S_{2.2 \mu \mathrm{m}}$. We cannot directly apply this extrapolation to our sample objects, since it applies only to the stellar $K$-band, and decompositions of the $K$-band continuum inside our aperture into stellar and AGN are usually not available. We have verified, however, that the conclusions reached in Sect. 3 are robust to stellar continuum contributions. For this purpose, we have repeated as an extreme assumption our analysis after subtracting directly from the $6 \mu \mathrm{m}$ continuum obtained in the spectral decomposition 0.19 times the total $K$-band continuum in an ISOPHOT-S aperture, which can be extrapolated with modest uncertainty from 2MASS data accessible in NED. The results given in Table 2 did not change significantly. We did not adopt these values, however, since they imply a systematic overcorrection for the many AGN dominated objects. Instead, we mark in Table 1 the few cases where a strong stellar contribution to our measured $6 \mu \mathrm{m}$ continuum is likely.

We do not attempt a correction of the mid-infrared fluxes for foreground extinction. Published extinction values for those objects refer to different tracers, and their applicability to the region dominating the mid-infrared flux is uncertain. Since the extinction at $6 \mu \mathrm{m}$ is $1 / 20$ or less of the visual extinction, typical $A_{\mathrm{V}}$ values from optical studies are irrelevant in any case. In the discussion of individual objects in the Appendix, we also mention NGC 6240 and NGC 4945 where obscuration of the mid-infrared AGN continuum is likely significant.

\subsection{X-ray emission}

We have compiled the $\mathrm{X}$-ray fluxes in the $2-10 \mathrm{keV}$ range from various literature sources using data from different satellites (e.g. ASCA, BeppoSAX, Chandra, XMM). For Seyfert 2s for which the absorbing column density $N_{\mathrm{H}}$ could be measured (most cases), the authors generally provide reliable measurements of the instrinsic, absorption-corrected hard X-ray flux. The intrinsic X-ray flux cannot be recovered when only a lower limit to $N_{\mathrm{H}}$ is inferred; this is the case for totally Compton thick 

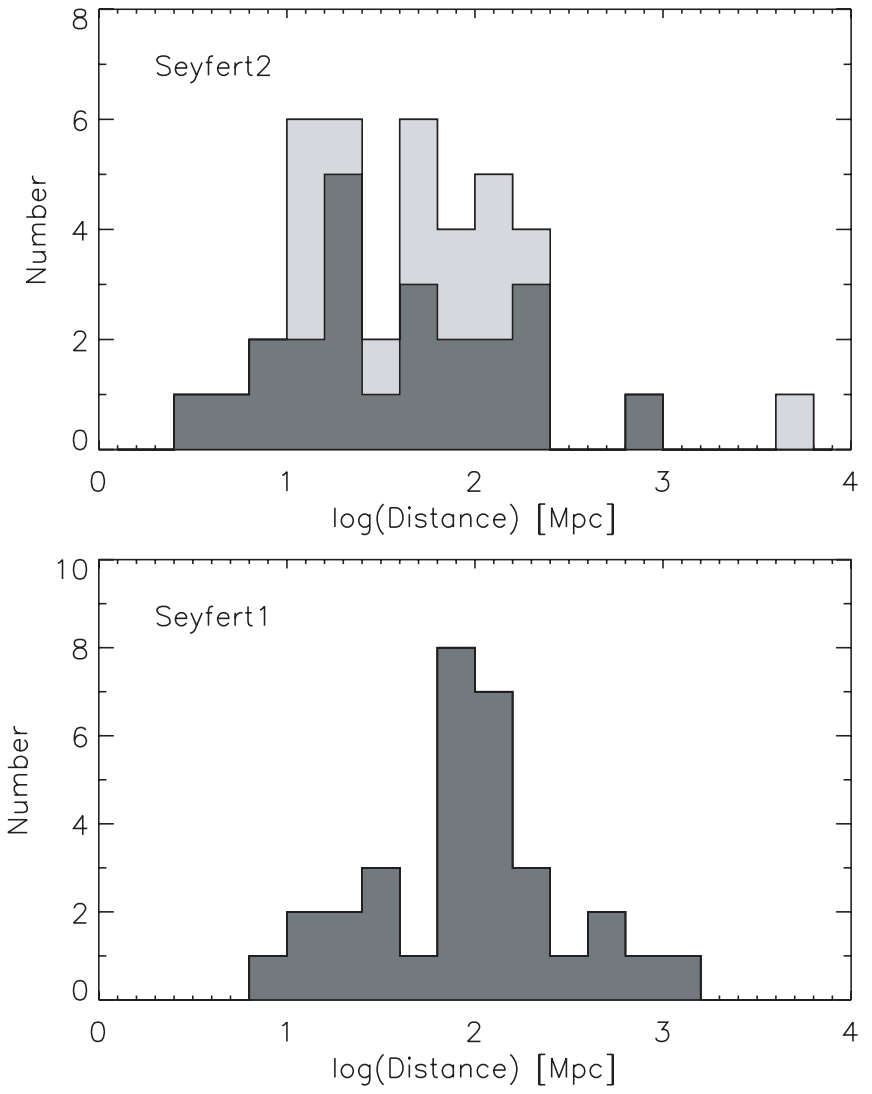

Fig. 2. Distance distribution for the objects in our sample, separated by type 1 ( 1 to 1.5 ) and 2 (1.8 to 2 ) Seyferts. Light shading identifies those Seyfert 2's where no absorption correction to the AGN hard $\mathrm{X}$-ray emission was possible. We adopted distances derived assuming $H_{0}=75, q_{0}=0.5$ except for M $51(8.4 \mathrm{Mpc}), \mathrm{M} 81(3.63 \mathrm{Mpc})$ and Circinus (4 Mpc).

nuclei with $N_{\mathrm{H}}>10^{25} \mathrm{~cm}^{-2}$, which are absorbed at all energies, or for mildly Compton thick nuclei with $N_{\mathrm{H}}>10^{24} \mathrm{~cm}^{-2}$ which are lacking data at $E>10 \mathrm{keV}$, i.e. for which any transmitted flux at high energy cannot be probed. For Seyfert 1 galaxies without published X-ray column we have assumed that observed and intrinsic flux can be safely adopted to be equal because of typically low absorbing columns. Figure 2 shows the distance distributions both for the full sample and for the subsample with absorption corrected X-ray fluxes.

\section{Results}

The observed mid-infrared and hard X-ray fluxes are listed in Table 1 and summarized in Fig. 3. The diagram shows the expected separation between type 1 and type 2 Seyferts, hard $\mathrm{X}$-ray emission in the latter often being strongly absorbed. More interesting is the comparison with absorption corrected X-ray fluxes shown in Fig. 4. A correlation between hard $\mathrm{X}$-rays and infrared continuum is indicated in this diagram but with considerable scatter that is not due to observational error in the mid-IR spectra. There is no offset between type 1 and 2 objects any more. Figure 5 compares mid-infrared and X-ray luminosities. Hard X-ray and mid-infrared luminosity correlate over four orders of magnitude in luminosity, the ratio of the two

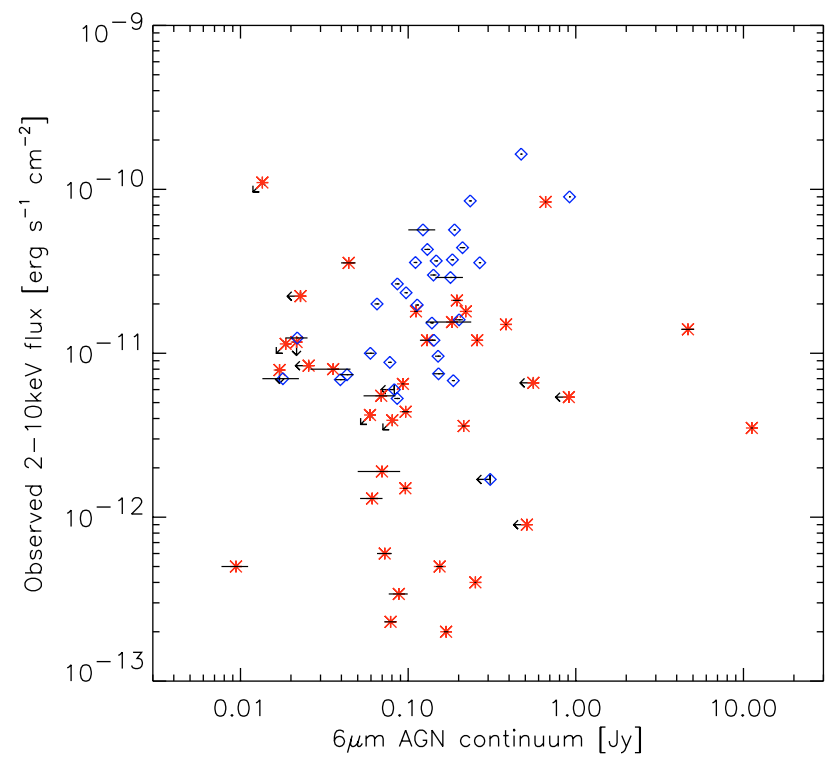

Fig. 3. Observed hard X-ray fluxes vs. $6 \mu \mathrm{m}$ AGN continuum for the sample galaxies. Diamonds indicate type 1 Seyferts (including objects classified as types $1,1.2,1.5$ ), asterisks indicate type 2 Seyferts (including objects classified as types $1.8,1.9,2$ ). Thin horizontal lines, in most cases smaller than the symbols, indicate the $1 \sigma$ uncertainty of the $6 \mu \mathrm{m}$ detections.

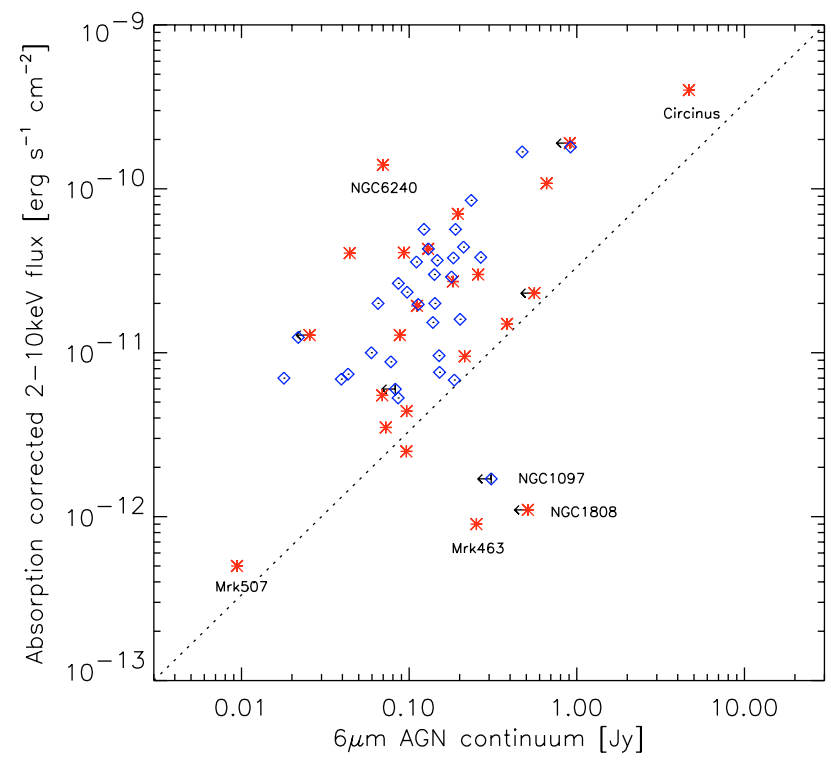

Fig. 4. Hard X-ray fluxes corrected for absorption vs. $6 \mu \mathrm{m}$ AGN continuum for those objects where an absorption corrected X-ray flux was available. Symbols are as in Fig. 3. The dotted line indicates slope 1, it is not a fit.

shows no clear trend with luminosity. The data are consistent with the same mean ratio, with considerable dispersion, over the entire range of luminosity.

\subsection{The scatter in the relation between mid-infrared continuum and hard $X$-ray emission}

Figure 6 shows histograms for the ratio of hard X-ray flux and mid-infrared AGN continuum, separated by Seyfert type. 


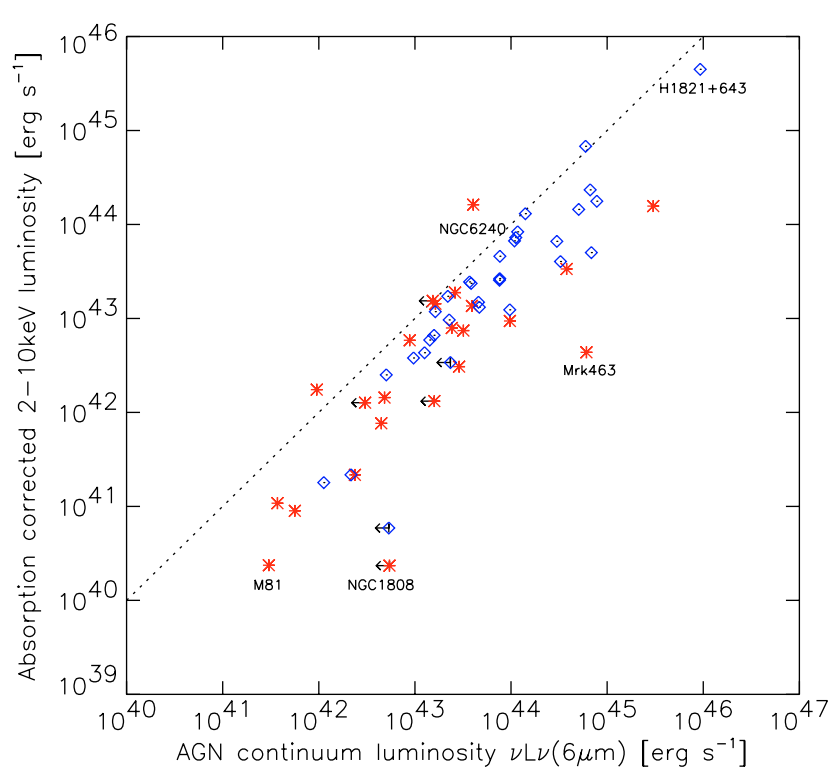

Fig. 5. Hard X-ray luminosities corrected for absorption vs. $6 \mu \mathrm{m}$ AGN continuum luminosities for those objects where an absorption corrected X-ray flux was available. Symbols are as in Fig. 3. The dotted line indicates slope 1 , it is not a fit.

The dispersion is considerable, the ratio for Seyfert $1 \mathrm{~s}$ varying over an order of magnitude. Ratios for type 2 Seyferts scatter yet wider. This may be partly due to inaccuracies in the absorption correction in the X-ray data. The current data do not allow to determine with certainty whether there is in reality a larger spread for type 2 than for type 1 . The two outlying objects Mrk 463 and NGC 6240 are briefly discussed in the Appendix. The spread observed in our sample is significantly larger than in the imaging study of Krabbe et al. (2001), where with the exception of the outlier NGC 6240 the ratio of intrinsic hard-X and mid-IR emission varies by just a factor of 3-4. This was probably a fortuitious effect of the small sample including $\sim 10$ times less objects than the present study. Given the many factors of AGN spectral energy distribution and geometry relating the hard X-ray flux and the dust reradiation of AGN emission in the mid-infrared, the larger scatter is not surprising. Another significant contribution to the spread is likely due to AGN variability. The X-ray and infrared measurements summarized in Table 1 were usually taken several years apart. Even in case of simultaneous observations, short term variability of the central engine may contribute to the scatter because of different time response and time averaging effects for the $\mathrm{X}$-rays and for the larger scale dust.

\subsection{No significant difference between Seyfert 1 and Seyfert 2 types}

A surprising result given the reasonable statistics of the present sample is the failure to detect the difference between type 1 and type 2 Seyferts that is predicted by the most straightforward versions of unified models. The median $\log \left(F(2-10 \mathrm{keV}) / v F_{v}(6 \mu \mathrm{m})\right)$ is -0.41 for type 1 objects and -0.63 for type 2 . This would not change strongly replacing the few lower limits by detections (Fig. 6). The key point
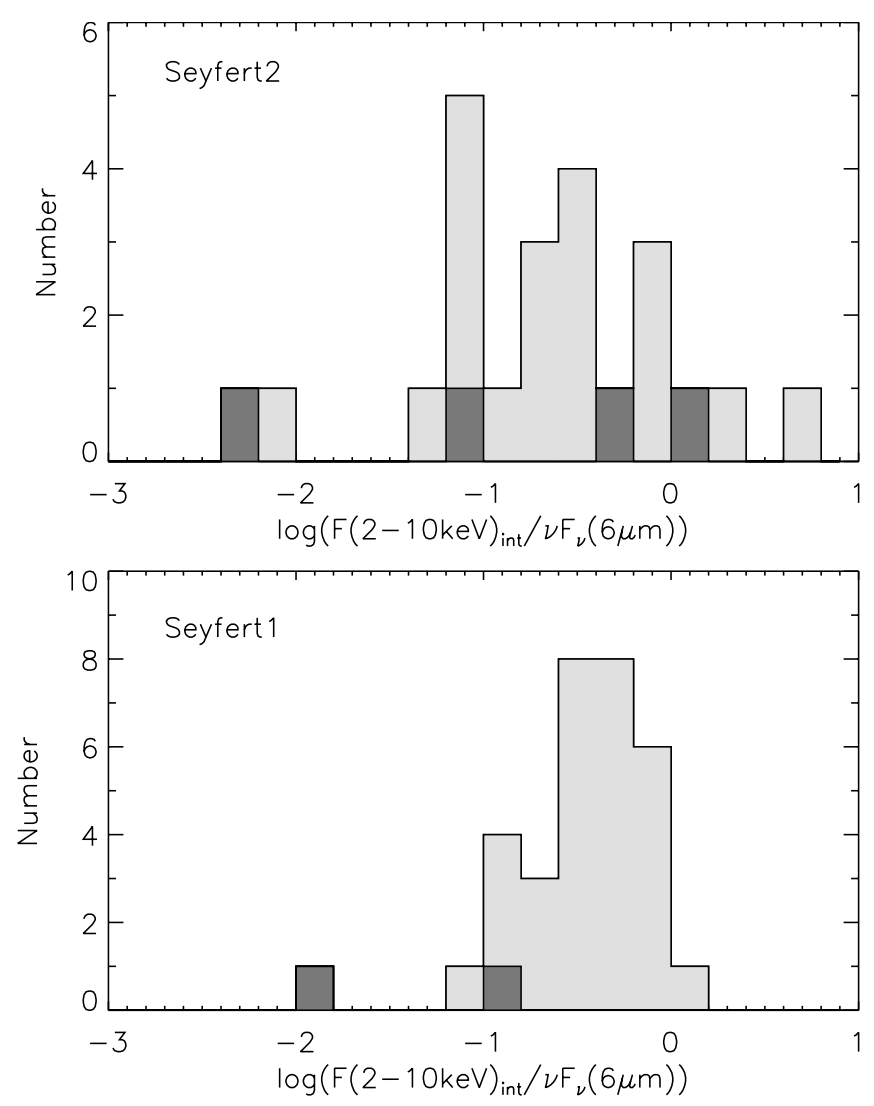

Fig. 6. Histograms showing for the two Seyfert types the logarithm of the ratio between intrinsic hard X-ray flux and $6 \mu \mathrm{m}$ AGN continuum. Dark shadings indicates objects with upper limits for the $6 \mu \mathrm{m}$ continuum, i.e. lower limits for the ratio shown.

is not the small difference found, which might even be reduced slightly if some of the Sy 2 limits were replaced by detections or with full correction for stellar continuum, it is the failure to detect a strong and significant difference in the opposite direction. If the mid-infrared continuum in Seyfert $2 \mathrm{~s}$ is suppressed by a factor of $\sim 8$ (Clavel et al. 2000), the hard X/IR ratio should be higher by the same factor compared to Seyfert $1 \mathrm{~s}$. While our targets are from a heterogeneous set of ISO observing programs, they do not represent a preselection by mid-IR flux which may affect such a comparison. The main observing programs involved did not invoke such a selection. A major part, e.g., is formed by objects from the CfA sample (Huchra \& Burg 1992). Also, for example, most AGN from the hard X-ray selected sample of Piccinotti et al. (1982) are included. As discussed by Maiolino \& Rieke (1995), samples like the CfA one may be biased against obscured objects and thus not reproduce the real fractions of Seyfert types. Our analysis normalizing to intrinsic X-rays should be robust to such effects as long as reaching lower but still significant numbers of obscured systems.

In unified schemes (e.g. Antonucci et al. 1993), the difference between Seyfert types is due to effects of viewing intrinsically similar objects from different directions, because an anisotropic distribution of absorbing material (e.g. the "torus") absorbs, scatters, and reprocesses the direct AGN light. In the most simple version, a central very small source (also emitting 


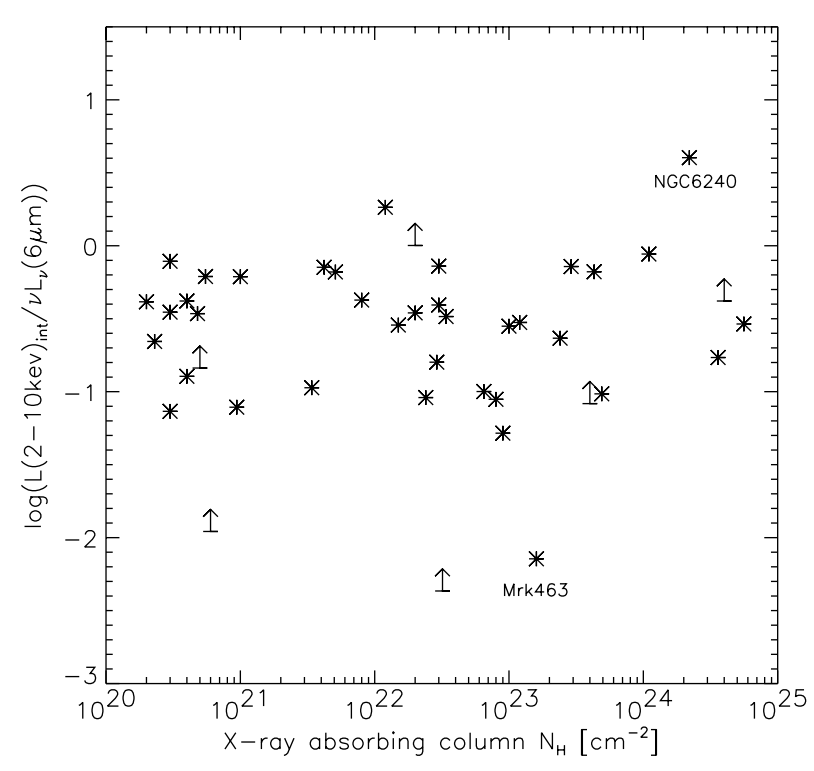

Fig. 7. Ratio of intrinsic hard X-ray flux and $6 \mu \mathrm{m}$ AGN continuum, plotted as a function of X-ray absorbing column.

the X-rays) illuminates a torus-like geometrically and optically thick dusty structure. Radiative transfer models of the emission from a geometrically and optically thick torus (e.g. Pier et al. 1992; Efstathiou \& Rowan-Robinson 1995; Granato et al. 1997; Nenkova et al. 2002) predict a strong anisotropy of the mid-infrared emission. The unified model in this case clearly predicts for Seyfert 2 galaxies a higher ratio of absorption corrected X-ray emission and mid-infrared continuum.

In a simple unified scheme invoking a torus, the ratio of intrinsic hard X-ray to observed mid-IR emission will increase from a fully face-on to a fully edge-on view. The difference between type $1 \mathrm{~s}$ and type $2 \mathrm{~s}$ could hence be masked if the type $2 \mathrm{~s}$ in the sample were preferentially objects with still relatively low obscuring columns, likely seen at intermediate angles. While we had to exclude fully Compton-thick Sy 2 s from the comparison because of the impossibility to derive an absorption corrected X-ray flux, they form well below half of the Sy 2 sample ( 5 of 38 , more could be among the 11 type 2 objects where the absorbing column is undetermined or only a limit to the $2-10 \mathrm{keV}$ flux available). Abandoning for a moment the normalization to X-rays, we have compared the average ratio of $6 \mu \mathrm{m}$ AGN continuum to $6.2 \mu \mathrm{m}$ PAH feature for the Seyfert 2's without intrinsic X-ray flux in our sample and the ones where an intrinsic flux could be derived. Both using the measurements/limits in Table 1 and using the raw measurements, the median AGN continuum to PAH ratio is only insignificantly ( 20\%) smaller for the 16 type 2 objects without intrinsic X-ray flux. With all caveats of normalizing to the PAHs rather than to intrinsic X-rays, this tentatively indicates that in a complete Sy 2 sample including the fully Compton-thick objects the mid-infrared AGN continuum will not be much lower.

More insight can be obtained from plotting the ratio of $\mathrm{X}$-ray and mid-infrared emission versus the X-ray absorbing column (Fig. 7). Column densities above $10^{23} \mathrm{~cm}^{-2}$ are well populated but do not show the upturn in the hard X/Mid-IR ratio expected at high columns in the simple unified scheme. In a naive foreground screen the optical depth at $6 \mu \mathrm{m}$ exceeds one already below $10^{23} \mathrm{~cm}^{-2}$. Radiative transfer in different more realistic "torus" geometries (see the large range in the models cited above) will predict different magnitudes of this effect and place it at different column densities. For example, a strong and fairly sharp upturn at $N_{\mathrm{H}} \sim 6 \times 10^{23} \mathrm{~cm}^{-2}$ is expected for the best fitting tapered disk model 5 e of Efstathiou \& Rowan-Robinson (1995). Placing predictions of future torus models on a fully empirical diagram like Fig. 7 should provide a valuable test of such models that does not make direct assumptions on viewing angles. Observationally, population of the columns around and above $10^{24} \mathrm{~cm}^{-2}$ will be important to characterize the effect of missing the most absorbed objects in a sample like ours.

One possibility why we fail to observe the expected signature of a torus is that much of the mid-infrared emission is from a more extended region emitting isotropically, and overwhelming a possible anisotropic emission. Observations have shown this to be the case for the $10 \mu \mathrm{m}$ emission of NGC 1068 where at least two thirds of the emission comes from an extended region overlapping the Narrow Line Region (e.g., Cameron et al. 1993; Bock et al. 1998) rather than a compact parsecscale torus. A similar result (at least 27\% extended emission) has recently been found for NGC 4151 (Radomski et al. 2003). High resolution observations and mid-infrared interferometry with large telescopes should be able to test in the near future whether the same applies to other Seyferts. This scenario of (mostly) larger scale and isotropic AGN continuum at first glance contradicts the results of Clavel et al. (2000), however, who find in their large aperture ISOPHOT-S data a $\sim 8$ times lower AGN continuum to host PAH ratio in Seyfert $2 \mathrm{~s}$ compared to Seyfert 1s. They ascribed this to orientation effects in a unified scenario, of a magnitude significantly larger than suggested earlier by Heckman (1995) and Maiolino et al. (1995). Much of their result could be an AGN luminosity effect rather than an orientation effect, though. Some of the relevant diagnostics are shown in Figs. 8 to 10 and Table 2 for our sample which has a significant overlap with the Clavel et al. (2000) sample. The distributions of PAH luminosities for the two types are consistent (Fig. 8), but the caveat that only upper limits to the $6.2 \mu \mathrm{m}$ PAH are available for many of the strong continuum Sy 1s has to be noted. This result means there are no obvious differences in the host properties as probed by the PAH emission, although some differences (as suggested e.g. by Maiolino et al. 1995) might be hidden by the many PAH upper limits, or by sample and physical aperture size (distance) mismatch between the two types. We note that upper limits also apply to the 6.2 and $7.7 \mu \mathrm{m}$ PAH features in many objects presented by Clavel et al. (2000), rather than detections reported through their method of including only positive flux above the noisy continuum into their PAH fluxes. For our sample, both the mid-infrared continuum luminosities (Fig. 9) and the absorption corrected X-ray luminosities (Fig. 10) are larger in Seyfert 1s than in Seyfert 2s. Reflecting the luminosity differences, a difference is also seen in the distance distributions (Fig. 2). All that argues that a difference in PAH equivalent 
Table 2. Properties of the type 1 and type 2 objects. The numbers quoted refer to the combination of detections and limits (see Figs. 6 to 10 for the fraction of limits which is highest among the Sy 1 PAH luminosities). Distances refer to the full sample, all others to the part with extinction corrected hard X-ray data (32 type 1, 23 type 2).

\begin{tabular}{lrrrr}
\hline \hline Property & \multicolumn{2}{c}{ Seyfert 1} & \multicolumn{2}{c}{ Seyfert 2} \\
& Median & Disp. & Median & Disp. \\
\hline $\log ($ Distance $)[\mathrm{Mpc}]$ & 2.00 & 0.50 & 1.68 & 0.61 \\
$\log \left(6.2 \mu \mathrm{m}\right.$ PAH luminosity) $\left[\mathrm{erg} \mathrm{s}^{-1}\right]$ & $(41.88)$ & $(0.85)$ & 41.50 & 0.99 \\
$\log \left(\mathrm{AGN}\right.$ continuum luminosity $\left.v L_{v}(6 \mu \mathrm{m})\right)\left[\mathrm{erg} \mathrm{s}^{-1}\right]$ & 43.88 & 0.84 & 43.20 & 1.00 \\
$\log \left(\right.$ Extinction corrected 2-10 keV luminosity) $\left[\mathrm{erg} \mathrm{s}^{-1}\right]$ & 43.39 & 0.99 & 42.64 & 1.07 \\
$\log \left(\mathrm{F}(2-10 \mathrm{keV}) / \mu \mathrm{F}_{v}(6 \mu \mathrm{m})\right)$ & -0.41 & 0.40 & -0.63 & 0.69 \\
\hline
\end{tabular}
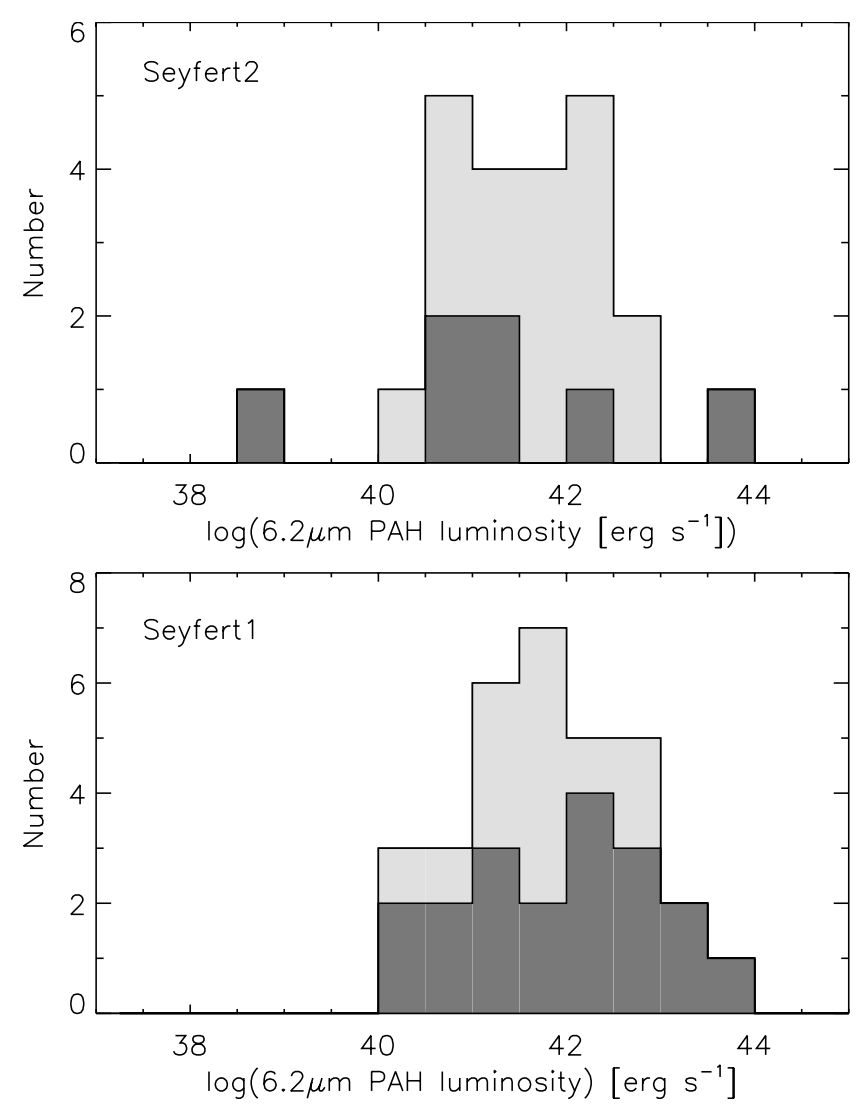

Fig. 8. Distribution of luminosities of the $6.2 \mu \mathrm{m}$ PAH emission feature. Dark shading indicates upper limits.

width between Seyfert 1s and 2s, found by Clavel et al. (2000), and also seen in our data (compare Fig. 8 and Fig. 9), can be largely due to the Seyfert 1 part of the samples representing both higher AGN luminosities and higher AGN/host luminosity ratios. This is a natural consequence of many AGN samples, including ones driving the selection of ISO observations, being biased in favour of more distant type 1 objects (see discussion in Maiolino \& Rieke 1995). Explaining our results by a dominant component of isotropic mid-infrared emission thus remains viable. We emphasise that a minor strongly anisotropic mid-infrared component may be present without affecting our analysis.

Another factor masking the signature of unification in the mid-IR to hard X-ray ratios could be uncertainties in the
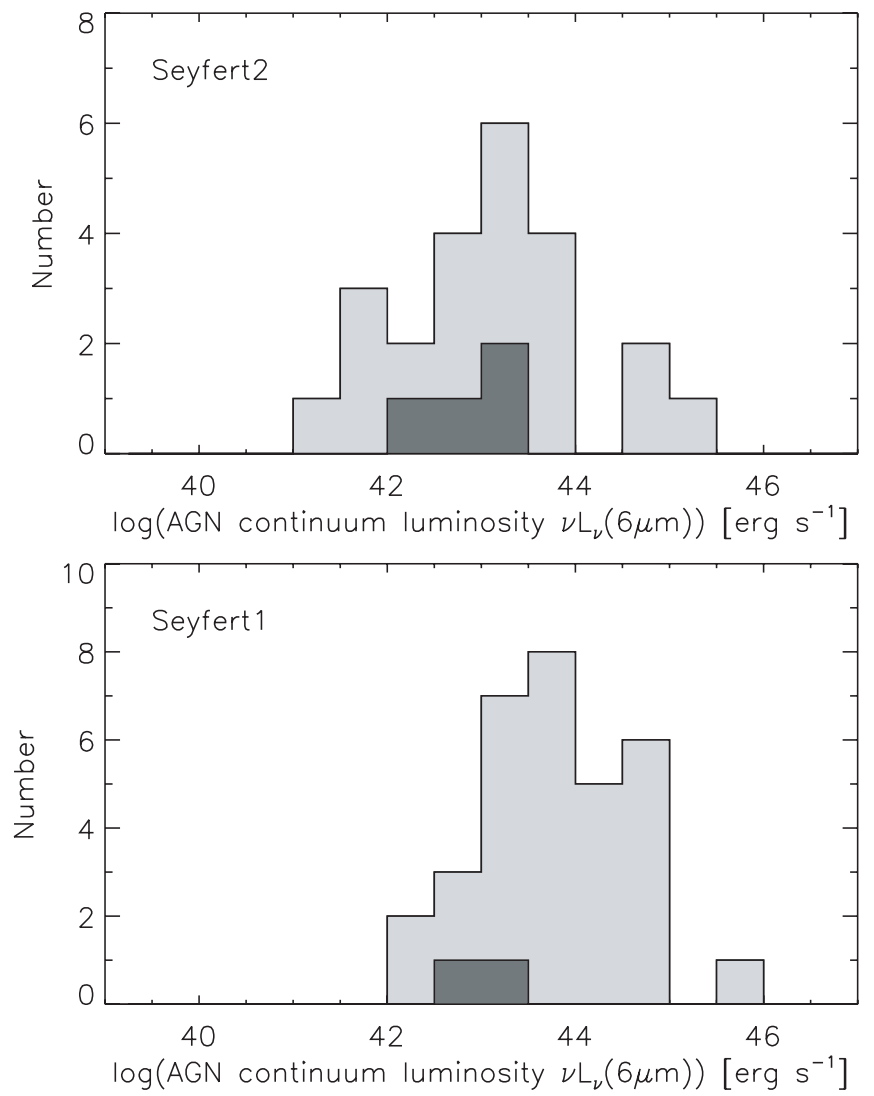

Fig. 9. Distribution of luminosities of the $6 \mu \mathrm{m}$ AGN continuum. Dark shading indicates upper limits.

absorption correction of the X-ray fluxes. While we have argued above that they may contribute to the increased scatter observed for the type 2 objects, we see no evidence for a systematic offset, which would also have profound implications on estimates of the energy budget of obscured X-ray sources. Similar arguments apply to scatter induced by intrinsic variations of the AGN spectral energy distributions between the hard X-rays and the wider spectral range that is heating dust. Finally, the strategy of our comparison relies on the assumption of isotropic X-ray emission (once considering the absorption corrections). If $\mathrm{X}$-rays were emitted anisotropically and if there were alignment between the structures causing this X-ray 


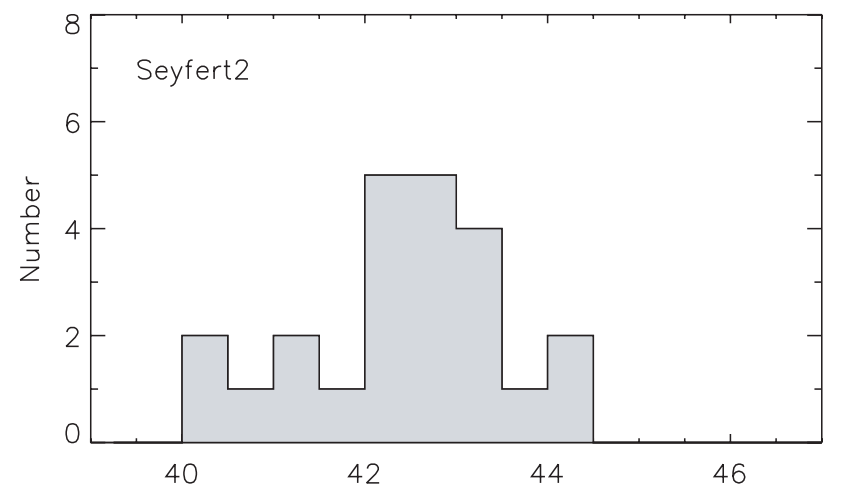

$\log$ (Absorption corrected 2-10keV luminosity) [ $\operatorname{erg~s}^{-1}$ ]

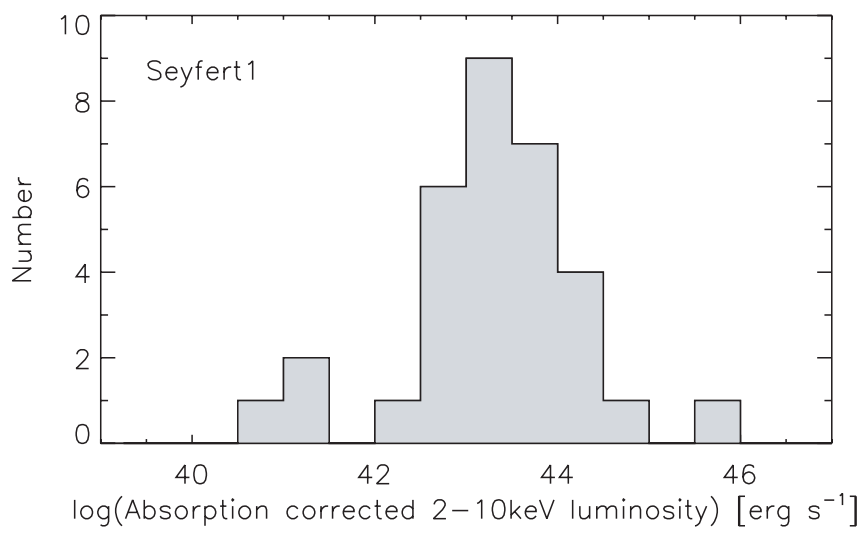

Fig. 10. Distribution of extinction corrected hard X-ray luminosities.

anisotropy and a torus absorbing/emitting anisotropically in the mid-IR, the expected difference in the ratio of hard $\mathrm{X}$ and midIR emission could cancel largely.

Our results suggest that local, mostly moderate luminosity, Seyferts do not show the behaviour expected if their midinfrared emission were dominated by a compact, anisotropically emitting torus. This adds to evidence that the most simple version of a unified scheme, in which all AGN at all luminosities are governed by such a structure, is not applicable. Recent deep X-ray and mid-IR surveys indicate significant differences in the evolution of high luminosity type 1 and lower luminosity type 2 populations that cannot be reconciled with a single type of obscuring structure in AGN of all luminosities and redshifts (e.g. Franceschini et al. 2002).

\section{Conclusions}

We have used spectral decomposition of a large sample of ISO spectra of AGNs to isolate the AGN $6 \mu \mathrm{m}$ continua. We compare these mid-infrared continua to intrinsic hard X-ray fluxes from the literature, assumed to be a fair isotropic measure of AGN luminosity. Due to this normalization, our comparison can test AGN properties and unification aspects without the level of sensitivity to selection biases (e.g. luminosities, ratio of type 1 and type 2 objects in the sample) that is found in comparisons of absolute quantitities or of normalizations to non-AGN quantitities. The main results are:

(1) Mid-infrared and intrinsic X-ray fluxes correlate, but with significant scatter. Ratios vary by more than an order of magnitude, the dispersion in the $\log$ is 0.4 for Seyfert 1 and 0.69 for Seyfert 2 galaxies. Main contributors to this spread likely include variations in the AGN spectral energy distribution and geometry of obscuring dust, as well as the effects of AGN variability.

(2) There is no significant difference between type 2 and type 1 in the average ratio of X-ray and mid-infrared continuum, in contrast to expectations from unified scenarios invoking an optically and geometrically thick torus emitting anisotropically in the mid-IR. Most likely, this is due to a large contribution from extended dust components emitting more isotropically. This will dilute anisotropic emission that, however, may still be present at lower levels.

Acknowledgements. We thank Sue Madden for information on the mid-IR properties of elliptical galaxies, the referee for helpful comments and Eckhard Sturm for discussions. We acknowledge support for the ISO spectrometer data center at MPE by DLR (50 QI 0202).

\section{Appendix A: Notes on selected objects}

NGC 1808 is believed to host both a strong spatially extended starburst (e.g. Krabbe et al. 1994) and possibly a weak and likely fading AGN (e.g. Bassani et al. 1999), although the $\mathrm{X}$-ray luminosity is low enough to overlap the regime of (also variable) non-AGN Ultraluminous X-ray Sources (ULX). Such objects are found at non-nuclear positions in other starbursting objects (Fabbiano et al. 2003). Because of the combination of strong star formation and weak AGN, the limit on AGN continuum is far off the correlation between mid-IR and X-rays.

NGC 1667 is likely another example of a fading "fossil" AGN (Bassani et al. 1999), introducing additional uncertainty on the AGN contribution at different wavelengths. For example, if the AGN is turning off the apparently high $N_{\mathrm{H}}$ may be an artifact of the reflected component remaining visible longer.

NGC 4945 The $6 \mu \mathrm{m}$ continuum is high compared to the average starburst, but not at the level of a significant detection of the AGN continuum given the scatter in starburst properties. The mid-IR weakness and corresponding location in our diagrams is certainly in part due to the foreground extinction which is high even in the mid-infrared in this system (Spoon et al. 2000). A high resolution mid-IR image, if possible outside the silicate band, is needed to break the ambiguity of both this result and the low resolution mid-IR imaging of Krabbe et al. (2001), and reach a mid-IR detection of the AGN.

NGC 6240 is one of the sources deviating most from the correlation, in the sense of high X-ray and low mid-IR. This could include a contribution of adopting a high estimate for the intrinsic X-ray emission (cf. also the lower estimate of Ikebe et al. 2000), and the likely noticeable obscuration of the $6 \mu \mathrm{m}$ continuum in this object. See Lutz et al. (2003) for a full discussion of the mid-infrared properties.

Mrk 463 has a very strong mid-IR AGN continuum but the relatively lowest absorption corrected hard X-ray emission compared to the average relation. The X-ray column may be underestimated. 


\section{References}

Alonso-Herrero, A., Quillen, A. C., Simpson, C., Efstathiou, A., \& Ward, M. J. 2001, AJ, 121, 1369

Antonucci, R. 1993, ARA\&A, 31, 473

Bassani, L., Dadina, M., Maiolino, R., et al. 1999, ApJS, 121, 473

Blustin, A. J., Branduardi-Raymont, G., Behar, E., et al. 2002, A\&A, 392,453

Bock, J. J., Marsh, K. A., Ressler, M. E., \& Werner, M. W. 1998, ApJ, 504, L5

Boller, T., Gallo, L. C., Lutz, D., \& Sturm, E. 2002, MNRAS, 336, 1143

Braito, V., Franceschini, A., Della Ceca, R., et al. 2003, A\&A, 398 , 107

Brandt, W. N., Fabian, A. C., Takahashi, K., et al. 1997, MNRAS, 290, 617

Cameron, M., Storey, J. W. V., Rotaciuc, V., et al. 1993, ApJ, 419, 136

Cappi, M., Bassani, L., Comastri, A., et al. 1999, A\&A, 344, 857

Clavel, J., Schulz, B., Altieri, B., et al. 2000, A\&A, 357, 839

Della Ceca, R., Pellegrini, S., Bassani, L., et al. 2001, A\&A, 375, 781

Della Ceca, R., Ballo, L., Tavecchio, F., et al. 2002, ApJ, 581, L9

Efstathiou, A., \& Rowan-Robinson, M. 1995, MNRAS, 273, 649

Fabbiano, G., Zezas, A., King, A. R., et al. 2003, ApJ, 584, L5

Franceschini, A., Braito, V., \& Fadda, D. 2002, MNRAS, 335, L51

Fukazawa, Y., Iyomoto, N., Kubota, A., Matsumoto, Y., \& Makishima, K. 2001, A\&A, 374, 73

Genzel, R., Weitzel, L., Tacconi-Garman, L. E., et al. 1995, ApJ, 444, 129

George, I. M., Turner, T. J., Yaqoob, T., et al. 2000, ApJ, 531, 52

Gondoin, P., Lumb, D., Siddiqui, H., Guainazzi, M., \& Schartel, N. 2001a, A\&A, 373, 805

Gondoin, P., Barr, P., Lumb, D., et al. 2001b, A\&A, 378, 806

Gondoin, P., Orr, A., Lumb, D., \& Santos-Lleo, M. 2002, A\&A, 388, 74

Gondoin, P., Orr, A., Lumb, D., \& Siddiqui, H. 2003a, A\&A, 397, 883

Gondoin, P., Orr, A., \& Lumb, D. 2003b, A\&A, 398, 967

Granato, G. L., Danese, L., \& Franceschini, A. 1997, ApJ, 486, 147

Guainazzi, M., Matt, G., Brandt, W. N., et al. 2000, A\&A, 356, 463

Guainazzi, M., Marshall, W., \& Parmar, A. N. 2001, MNRAS, 323, 75

Heckman, T. M. 1995, ApJ, 446, 101

Huchra, J., \& Burg, R. 1992, ApJ, 393, 90

Ikebe, Y., Leighly, K., Tanaka, Y., et al. 2000, MNRAS, 316, 433

Immler, S., Brandt, W. N., Vignali, C., et al. 2003, AJ, 126, 153

Iyomoto, N., Makishima, K., Fukazawa, Y., et al. 1996, PASJ, 48, 231

Krabbe, A., Sternberg, A., \& Genzel, R. 1994, ApJ, 425, 72

Krabbe, A., Böker, T., \& Maiolino, R. 2001, ApJ, 557, 626

Landi, R., Bassani, L., Malaguti, G., et al. 2001, A\&A, 379, 46

Laurent, O., Mirabel, I. F., Charmandaris, V., et al. 2000, A\&A, 359, 887

Lawson, A. J., \& Turner, M. J. L. 1997, MNRAS, 288, 920
Lu, N., Helou, G., Werner, M. W., et al. 2003, ApJ, 588, 199

Lutz, D., Sturm, E., Genzel, R., et al. 2003, A\&A, 409, 867

Maiolino, R., Ruiz, M., Rieke, G. H., \& Keller, L. D. 1995, ApJ, 446, 561

Maiolino, R., \& Rieke, G. H. 1995, ApJ, 454, 95

Maiolino, R., Salvati, M., Bassani, L., et al. 1998, A\&A, 338, 781

Malizia, A., Bassani, L., Stephen, J. B., Malaguti, G., \& Palumbo, G. G. C. 1997, ApJS, 113, 311

Malizia, A., Bassani, L., Zhang, S. N., et al. 1999, ApJ, 519, 637

Matt, G., Guainazzi, M., Frontera, F., et al. 1997, A\&A, 325, L13

Matt, G., Guainazzi, M., Maiolino, R., et al. 1999, A\&A, 341, L39

Matt, G., Perola, G. C., Fiore, F., et al. 2000, A\&A, 363, 863

Mazzarella, J., Voit, G. M., Soifer, B. T., et al. 1994, AJ, 107, 1274

Mirabel, I. F., Vigroux, L., Charmandaris, V., et al. 1998, A\&A, 333, L1

Nandra, K., \& Pounds, K. A. 1994, MNRAS, 268, 405

Nenkova, M., Ivezić, Z., \& Elitzur, M. 2002, ApJ, 570, L9

Perola, G. C., Matt, G., Fiore, F., et al. 2000, A\&A, 358, 117

Perola, G. C., Matt, G., Cappi, M., et al. 2002, A\&A, 389, 802

Piccinotti, G., Mushotzky, R. F., Boldt, E. A., et al. 1982, ApJ, 253, 485

Pier, E. A., \& Krolik, J. H. 1992, ApJ, 401, 99

Polletta, M., Bassani, L., Malaguti, G., Palumbo, G. G. C., \& Caroli, E. 1996, ApJS, 106, 399

O’Brien, P. T., Page, K., Reeves, J. N., et al. 2001, MNRAS, 327, L37

Ogasaka, Y., Inoue, H., Brandt, W. N., et al. 1997, PASJ, 49, 179

Radomski, J. T., Piña, R. K., Packham, C., et al. 2003, ApJ, 587, 117

Rao, A. R., Singh, K. P., \& Vahia, M. N. 1992, MNRAS, 255, 197

Reeves, J. N., \& Turner, M. J. L. 2000, MNRAS, 316, 234

Reynolds, C. S. 1997, MNRAS, 286, 513

Risaliti, G., Maiolino, R., \& Bassani, L. 2000, A\&A, 356, 33

Severgnini, P., Risaliti, G., Marconi, A., Maiolino, R., \& Salvati, M. 2001, A\&A, 368, 44

Spinoglio, L., Malkan, M. A., Rush, B., Carrasco, L., \& Recillas-Cruz, E. 1995, ApJ, 453, 616

Spoon, H. W. W., Koornneef, J., Moorwood, A. F. M., Lutz, D., \& Tielens, A. G. G. M. 2000, A\&A, 357, 898

Spoon, H. W. W., Keane, J. V., Tielens, A. G. G. M., et al. 2002, A\&A, 385,1022

Spoon, H. W. W., Moorwood, A. F. M., Lutz, D., et al. 2004, A\&A, 414,873

Sturm, E., Lutz, D., Tran, D., et al. 2000, A\&A, 358, 481

Thuan, T. X., Sauvage, M., \& Madden, S. 1999, ApJ, 516, 783

Tran, Q. D., Lutz, D., Genzel, R., et al. 2001, ApJ, 552, 527

Turner, T. J., Romano, P., George, I. M., et al. 2001, ApJ, 561, 131

Vignati, P., Molendi, S., Matt, G., et al. 1999, A\&A, 349, L57

Xilouris, E. M., Madden, S. C., Galliano, F., Vigroux, L., \& Sauvage, M. 2004, A\&A, 416, 41

Yang, Y., Wilson, A. S., \& Ferruit, P. 2001, ApJ, 563, 124 